\section{Strangulated umbilical hernia after esophagogas- troduodenoscopy in a patient with liver cirrhosis and ascites}

Umbilical hernias are present in up to $20 \%$ of patients with long-standing cirrhosis and $40 \%$ of those with ascites. There are some case reports of incarcerated umbilical and diaphragmatic hernias following colonoscopy. To our knowledge, this is only the second case of umbilical hernia strangulation following esophagogastroduodenoscopy (EGD) in the literature [1]. A 46-year-old white man with a history of decompensated alcoholic liver disease, esophageal varices, refractory ascites, and umbilical hernia underwent elective outpatient EGD under conscious sedation for variceal surveillance. Band ligation was performed with no complications. The patient tolerated the procedure well and was released on the same day. The next day he presented to the gastroenterology clinic with complaints of abdominal pain, nausea, vomiting, and irreducible painful umbilical hernia. He had neither had a bowel movement nor passed flatus since the procedure. Emergent abdominal radiography ( $\bullet$ Fig. 1 ) showed smallbowel obstruction. The patient underwent emergent exploratory laparotomy which revealed a small umbilical defect with a strangulated loop of small bowel, which was resected with primary sideto-side stapled anastomosis along with umbilical hernia repair via conventional fascial technique.

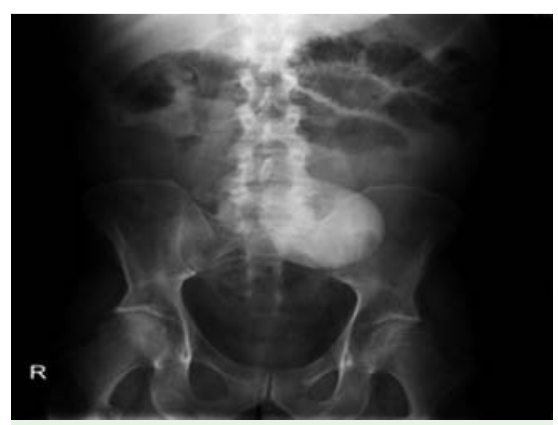

Fig. 1 Abdominal radiograph showing an umbilical hernia as a well-circumscribed soft tissue mass and small-bowel dilatation.

This case demonstrates hernia incarceration and strangulation as an extremely rare complication following EGD. The probable cause was the increased intraabdominal pressure secondary to air insufflation during the procedure, which would have led to dilation of small bowel loops, thus precipitating the incarceration. It is very unlikely that the timing of this complication was a coincidence, given the chronological order of events and that the patient had this hernia for a long time with no complications.

Endoscopy_UCTN_Code_CPL_1AH_2AK

Competing interests: None

\section{Hazem T. Hammad, Venu Chen- namaneni, Dina S. Ahmad, Mohammad Esmadi}

Department of Internal Medicine, Division of Gastroenterology and Hepatology, Columbia, Missouri, USA

\section{References}

1 Bynum TE, Smalley TK. Rupture of ascitic umbilical hernia during esophagoscopy; an unusual indirect complication. Gastrointest Endosc 1970; 17: 67-68

Bibliography

Dol http://dx.doi.org/

10.1055/s-0034-1364883

Endoscopy 2014; 46: E247

(C) Georg Thieme Verlag KG

Stuttgart · New York

ISSN 0013-726X

Corresponding author

Mohammad Esmadi, MD

One Hospital Dr.

DC043.00 Health Sciences Center

Columbia

MO 65212

USA

Fax: +1-573-884-5690

mohsmadi@hotmail.com 